\title{
Leading baryons at ZEUS
}

\author{
Graziano Bruni
}

INFN - Bologna

ZEUS Collaboration 


\section{Outline}

- Introduction

- Recent results from HERAI data

- Leading protons $\left(12.8 \mathrm{pb}^{-1}\right)$

- Leading neutrons $\left(40 \mathrm{pb}^{-1}\right)$

- Comparison to models

- Dijets and leading neutrons in $y p\left(40 \mathrm{pb}^{-1}\right)$ 


\section{Semi-inclusive reaction}

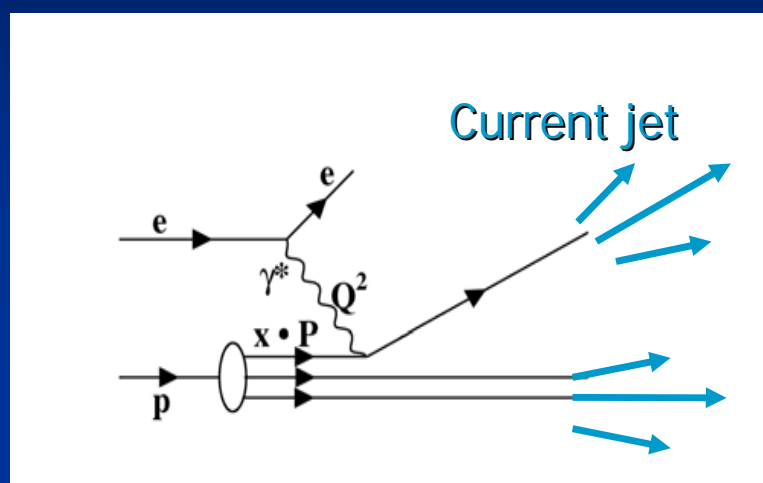

Proton remnant
$\operatorname{DIS}\left(\mathrm{Q}^{2}>1-2 \mathrm{GeV}^{2}\right)$

$$
e p \rightarrow e+L B+X
$$

Scale for secondary particle production decreases from Q2 (current region) to a soft hadronic scale (proton fragmentation region)

\section{Photoproduction - vp}

Hadronic component of the photon.

Can re-introduce hard scale (e.g. requiring high-pT jets)

\section{Production Models}
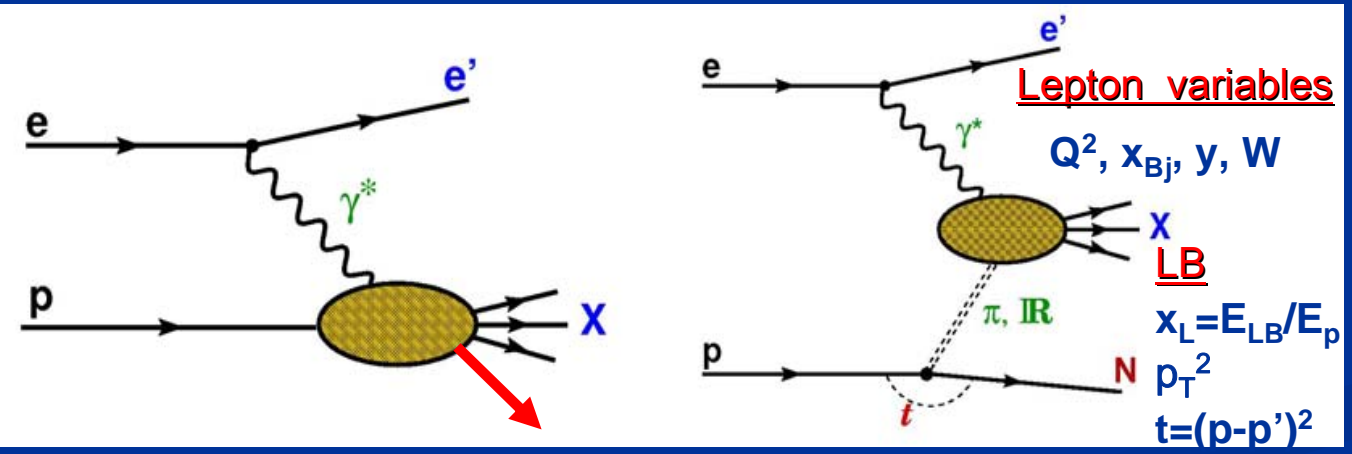

\begin{tabular}{l} 
MC fragmentation models \\
\hline - Cluster model (Herwig) \\
- MEPS, parton shower + SCI \\
- CDM (Ariadne) \\
Dynamical particle-exchange models \\
\hline - leading protons: $\pi_{0}, \mathrm{IR}, \mathrm{IP}$ \\
$\quad$ (isoscalar + isovector) \\
$\quad$ - leading neutrons: $\pi^{+}, \rho^{+}, \ldots$ \\
$\quad$ (isovector) \\
\hline
\end{tabular} 


\section{Rescattering and absorption}

D'Alesio and Pirner - "geometrical" picture (EPJ A7 (2000) 109)

e.g. LN production via p-exchange - Neutron absorption through rescattering

Absorption: neutron rescatters on $\gamma$ hadronic component (enhanced when $p$-n system size $r_{p n} \sim 1 / p_{T}$ is small w.r.to the $y$ transverse size).

Pion-flux: $\left\langle r_{\pi n}>\right.$ increases with $x_{L} \rightarrow$ more absorption at low $x_{L}$

- $n$ kicked to lower $x_{L} \&$ higer $p_{T} \rightarrow$ migration

- $\mathrm{n}$ may escape detection $\rightarrow$ absorption loss

$\square$ More absorption in photoproduction than DIS ( $\gamma$ transverse "size" larger)
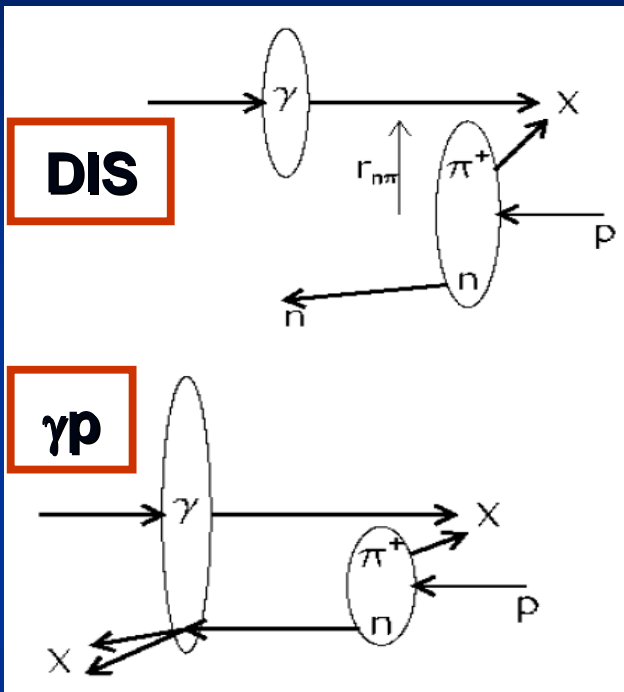

\section{Additional Corrections}

Nikolaev, Speth,Zakharov (NSZ), Kaidalov, Khoze, Martin, Ryskin (KKMR) (hep-ph/9708290, 0602215, 0606213)

$\square$ Corrections due to re-scattering processes via additional IP exchanges ( $\rightarrow$ Uncertainties in $\pi$ structure function extraction )

$\square$ Enhanced absorptive corrections, calculation of migrations, inclusion of additional exchanges ( $\rho$ and a2) ( $\rightarrow$ different $x_{L} \& p_{T}$ dependences)

$>$ Measure $\mathrm{x}_{\mathrm{L}}$ and $\mathrm{p}_{\mathrm{T}}^{2}$ dist.

$>$ Study $\mathrm{Q}^{2}$ dependence

$>$ Compare $\gamma p$ and DIS

$>$ Look for effects due to absorption 


\section{Main experimental tools}




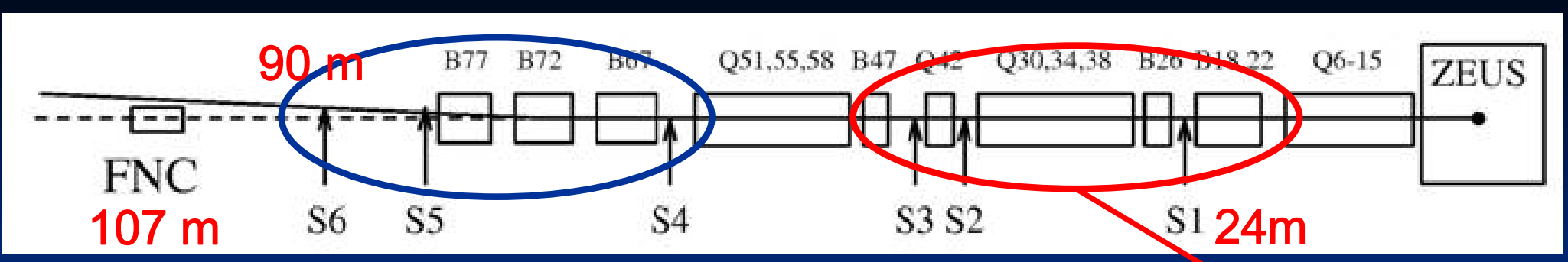

Lower $x_{L}$ accessible

\section{Forward Neutron Calorimeter + Tracker}

proton beam

acceptance window

$\Theta_{\mathrm{n}}<0.75 \mathrm{mrad}$

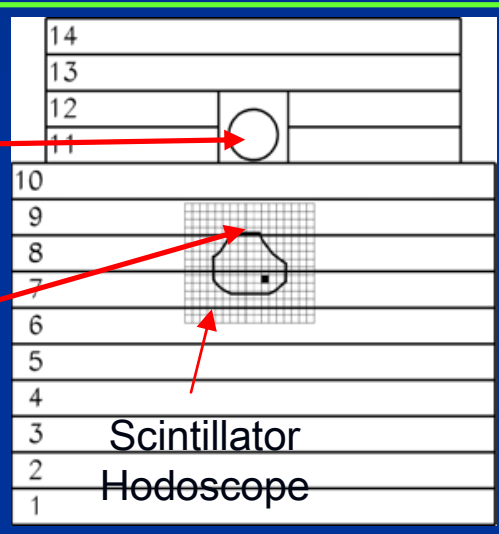

- FNC: 10ג lead-scintillator sandwich

- $\sigma / E=0.65 / \sqrt{E}$, Energy scale $2 \%$

- Acceptance $\theta_{n}<0.8 \mathrm{mrad}$, azimuthal coverage $30 \%$. For $x_{L}>0.6,<A>\sim 25 \%$.

- FNT: Scint. hodoscope @ $1 \lambda_{\text {int, }}$ $\sigma_{x, y}=0.23 \mathrm{~cm}, \sigma_{\theta}=22 \mu \mathrm{rad}$

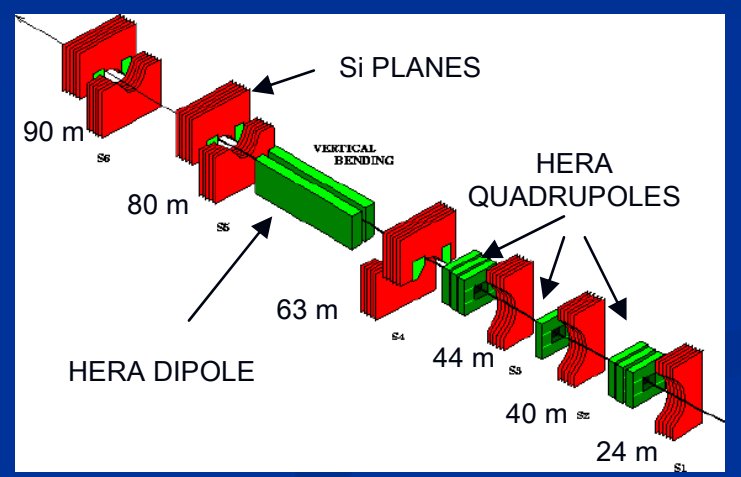

S1-S2-S3 and S4-S5-S6

Independent spectrometers Accepted $p_{T}$ varies with $x_{L}$ For $\mathrm{x}_{\mathrm{L}}>0.6, \mathrm{p}_{\mathrm{T}}^{2}<0.5 \mathrm{GeV}^{2}$, $<A>\sim 15 \%$

$$
\left.\mathrm{p}_{\mathrm{T}}{ }^{2} \text { resolution better than } \mathrm{p} \text {-beam spread ( } 50 \text { - } 100 \mathrm{MeV}\right)
$$


$x_{L}$ and $p_{T}{ }^{2}$ spectra 


\section{$x_{L}$ yields: normalized to inclusive DIS}

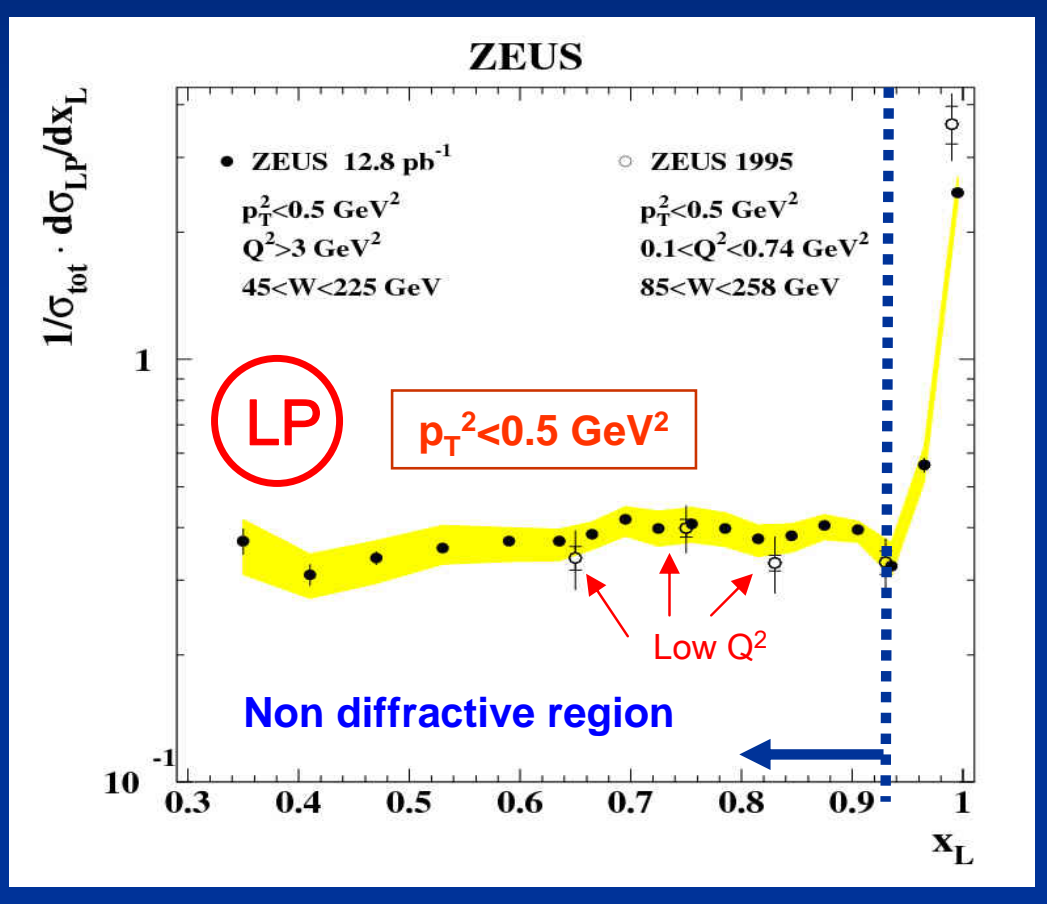

Yield flat below diffractive peak

DIS and earlier low $\mathrm{Q}^{2}$ data are compatible

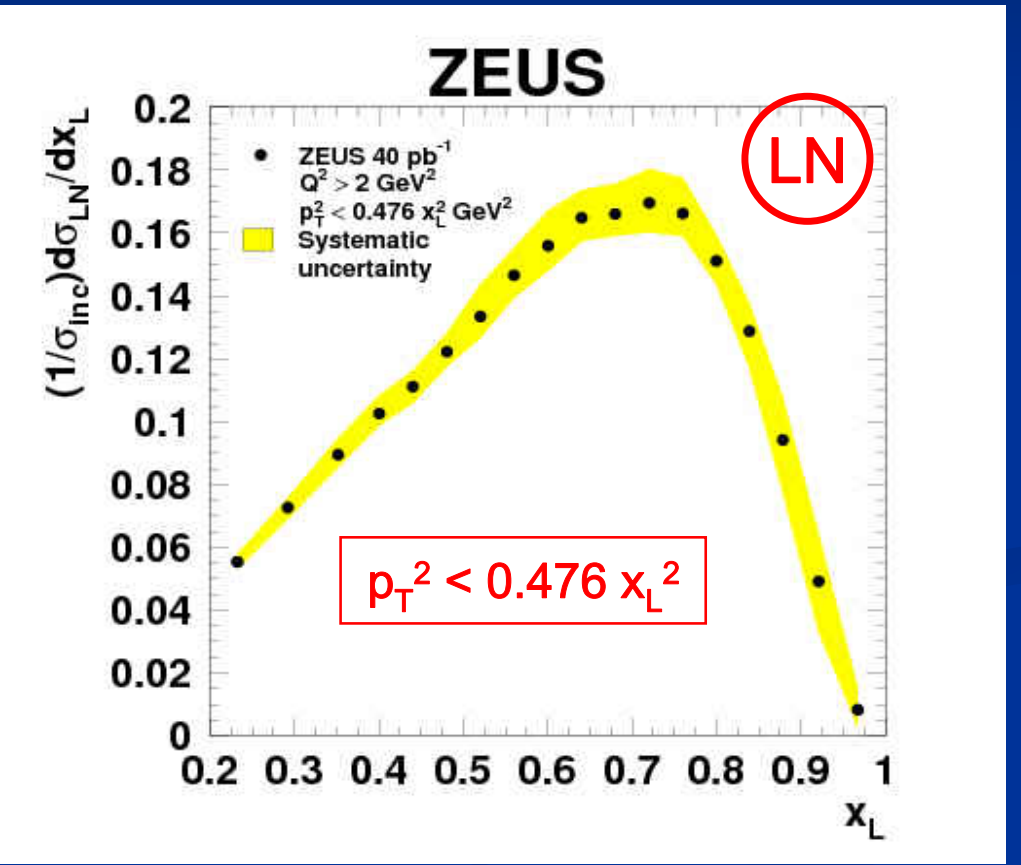

LN: Yield $\rightarrow 0$ at kinematic limit, $x_{L} \rightarrow 1$

Drop at $x_{L}<0.7$ due to drop in acceptance 


\section{$x_{L}$ - comparison LP, LN}

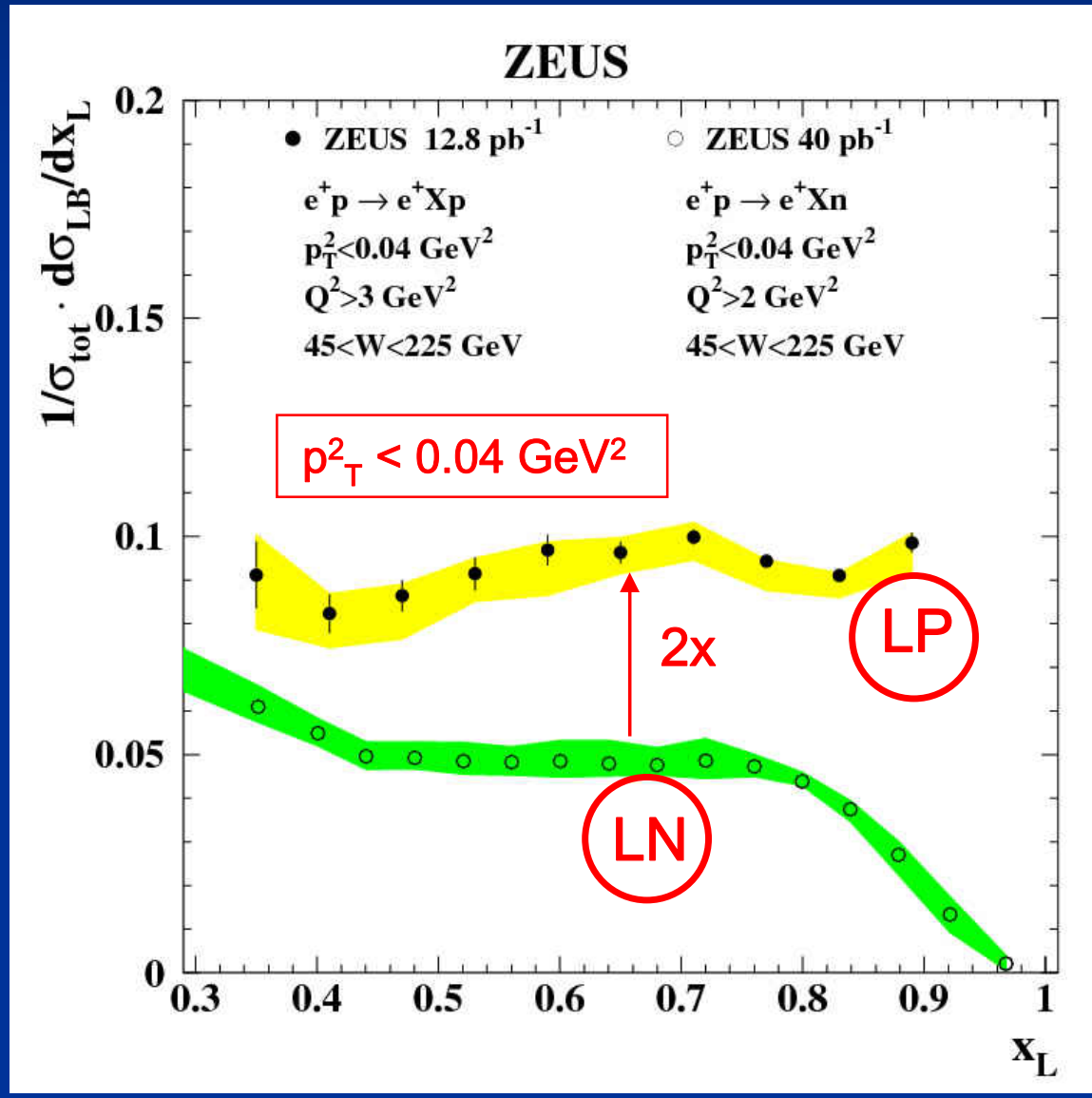

Same $p_{\mathrm{T}}^{2}$ range: $\mathrm{p}_{\mathrm{T}}^{2}<0.04 \mathrm{GeV}^{2}$

Pure isovector exchange (like pion)

$$
L N=2 L P
$$

Data show :

$$
\mathrm{LP} \sim 2 \mathrm{LN}
$$

$\Rightarrow$ Other isoscalar IR contributions needed to account for the LP rates 


\section{$\mathrm{P}_{\mathrm{T}}{ }^{2}$ distributions in $\mathrm{X}_{\mathrm{L}}$ bins}
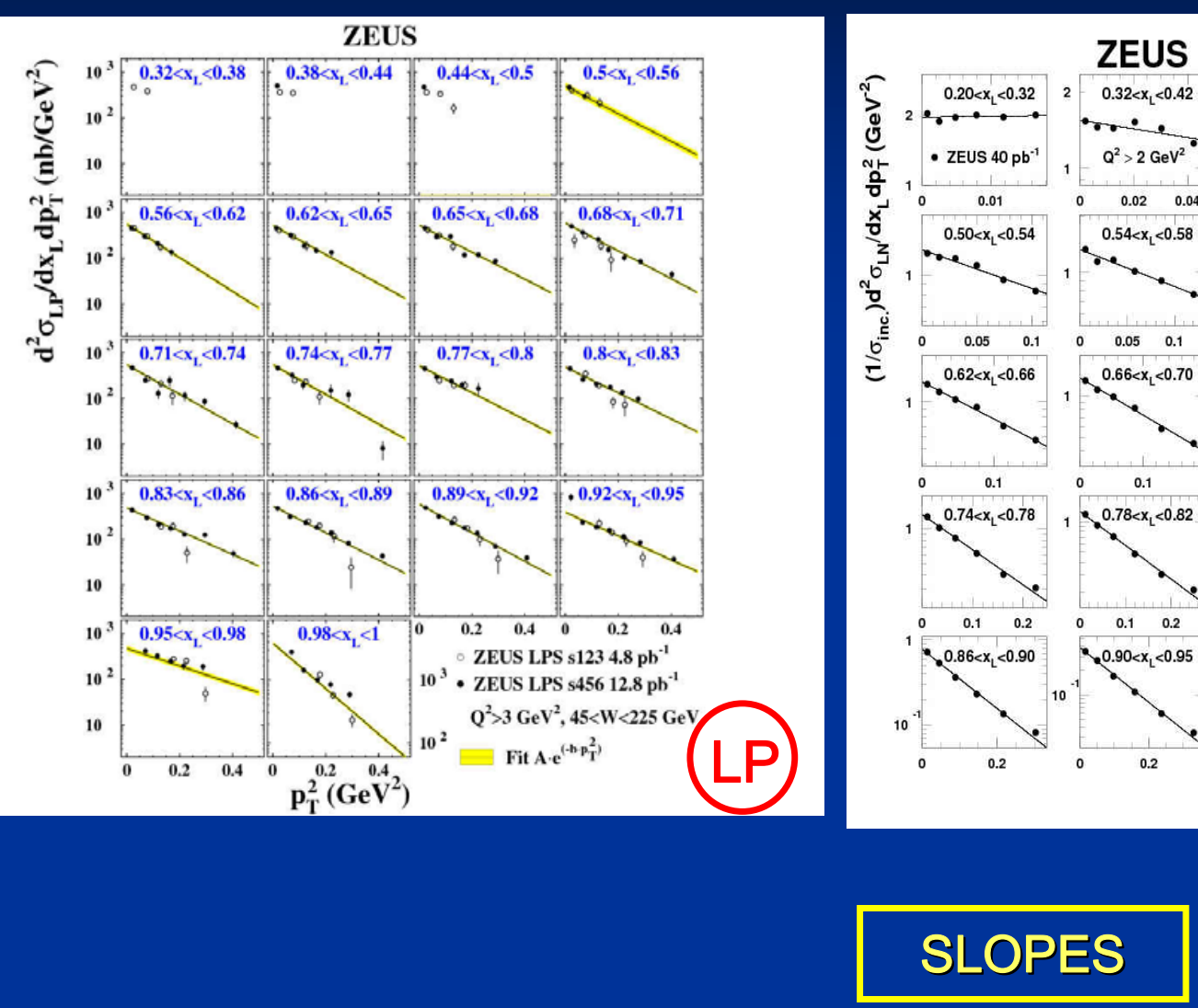

Different trend.

Similar around $x_{\mathrm{L}} \sim 0.7$

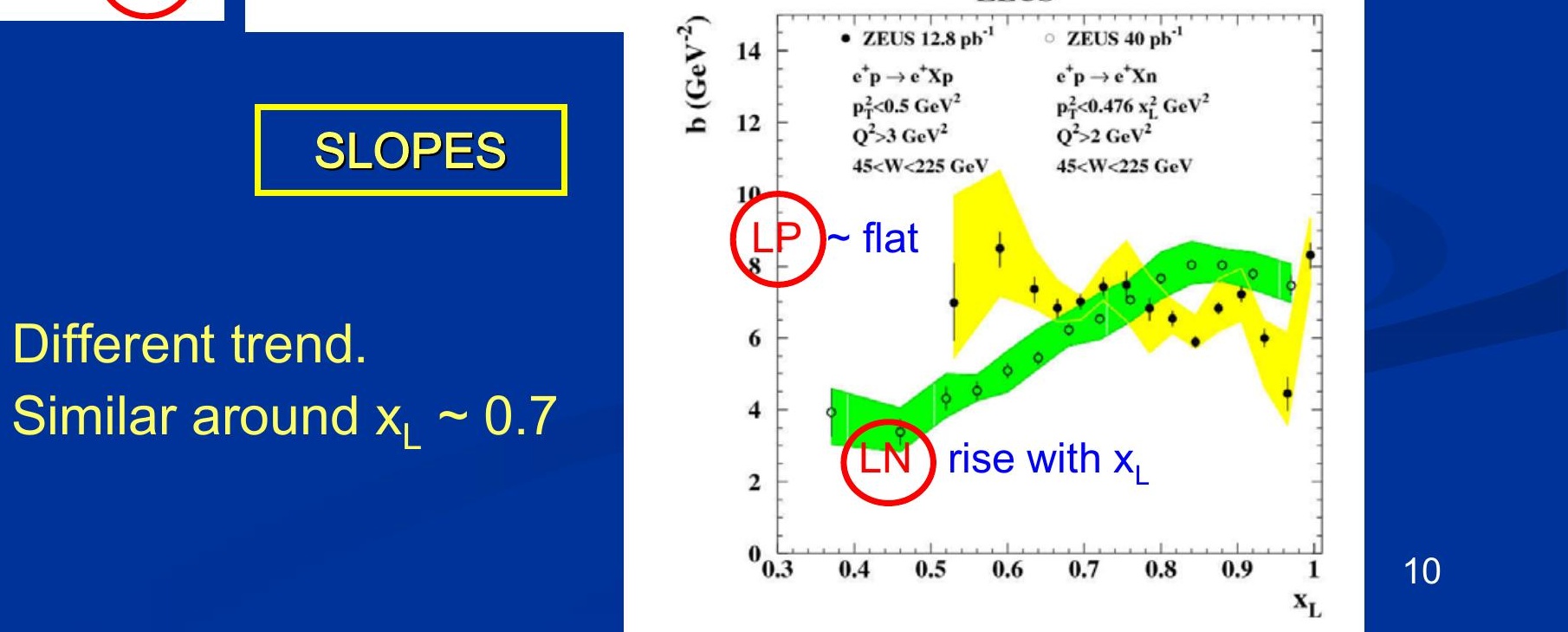

LN:

$\mathrm{p}_{\mathrm{T}}{ }^{2}$ range varies with $\mathrm{x}_{\mathrm{L}}$ bin

Exponential behaviour

$\frac{1}{\sigma_{i n c}} \frac{d^{2} \sigma_{L B}}{d x_{L} d p_{T}^{2}}=a\left(x_{L}\right) \cdot e^{-b\left(x_{L}\right) p_{T}^{2}}$

\section{ZEUS}




\section{$\mathrm{Q}^{2}$ dependence}




\section{LP \\ rates to inclusive DIS}

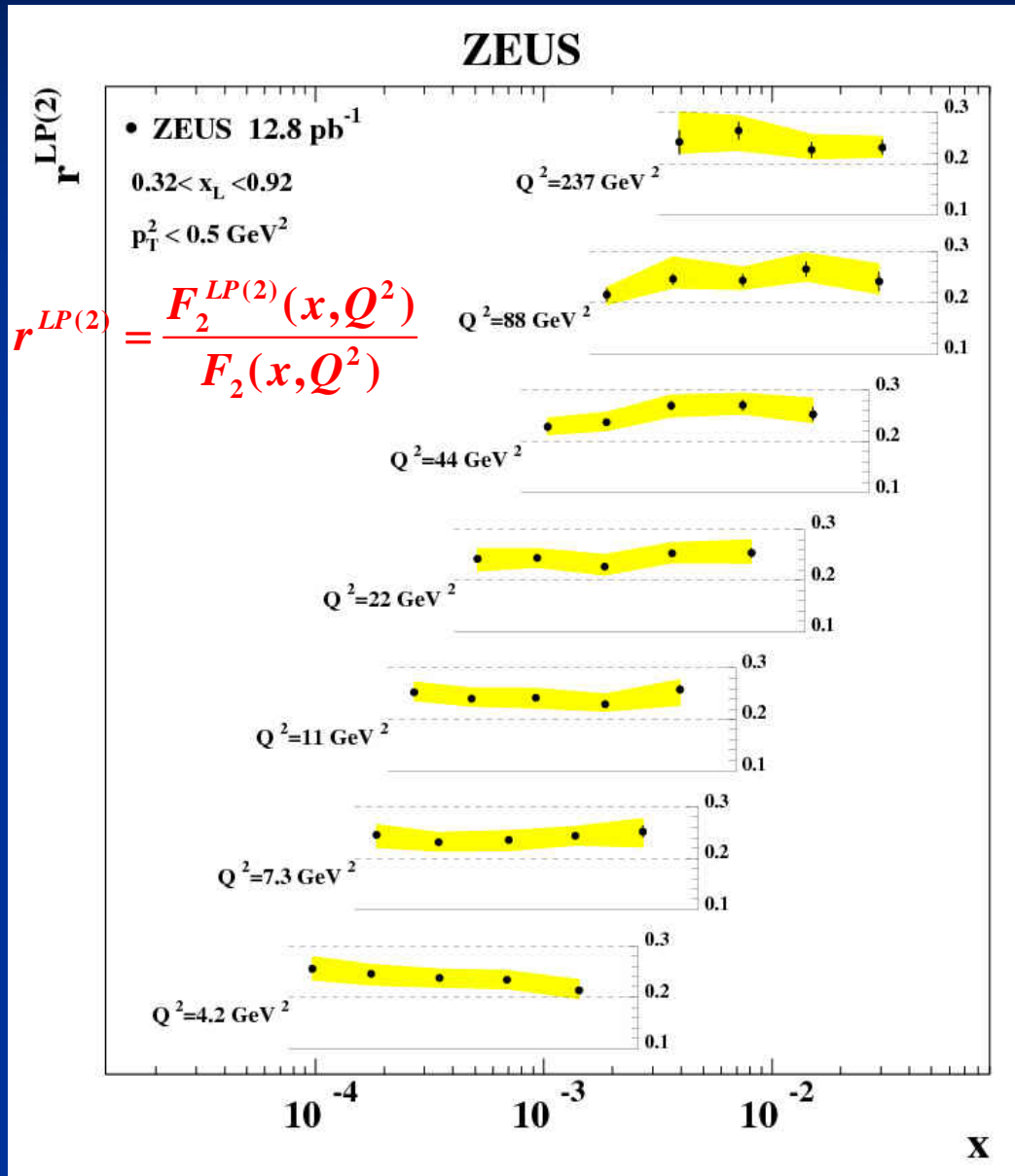

$\sim 24 \%$ of DIS events have a LP with $0.32<x_{L}<0.92$, almost independently of $x$ and $Q^{2}$

\section{Average LP yield vs $\mathrm{Q}^{2}$}

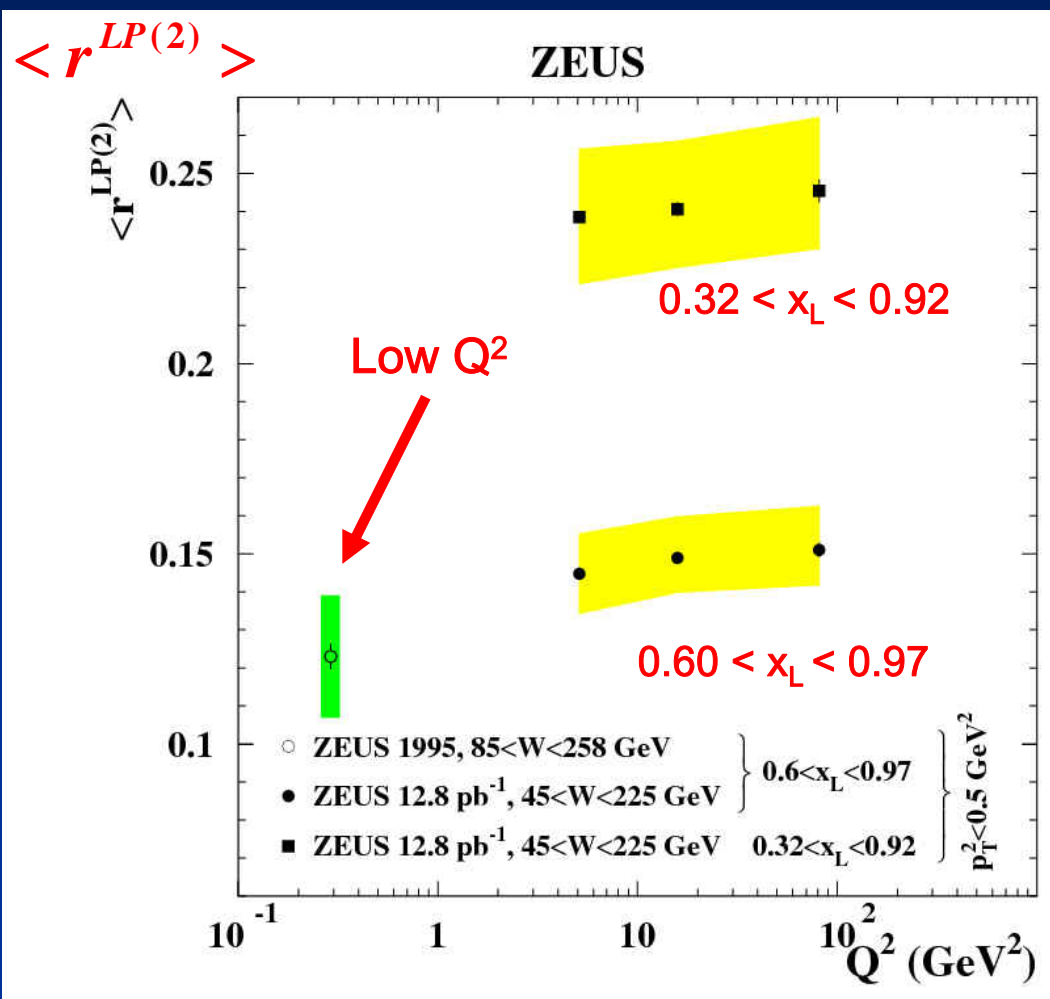

Slight decrease with decreasing $\mathrm{Q}^{2}$ (larger $\mathrm{Y}^{*}$ transverse size) not excluded

Consistent with absorptive effects 


\section{LN: $Q^{2}$ dependence of $x_{L}$ yields}

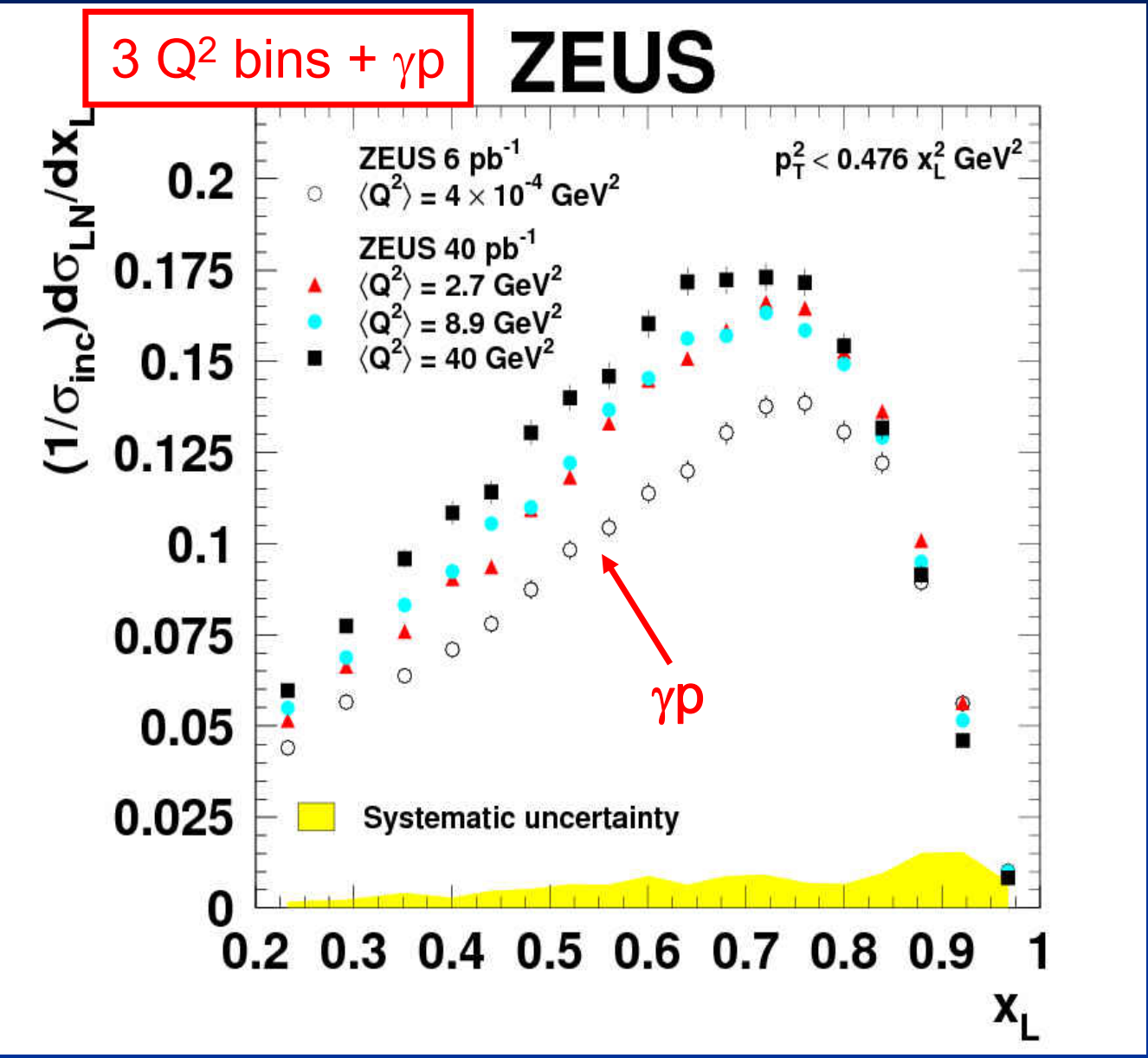

$>$ Yield decreases with decrease of $Q^{2}$ : large effect from DIS to $\gamma p$

$>$ Smaller $\mathrm{Q}^{2}$ dependence at intermediate values

$>$ Violation of vertex factorization

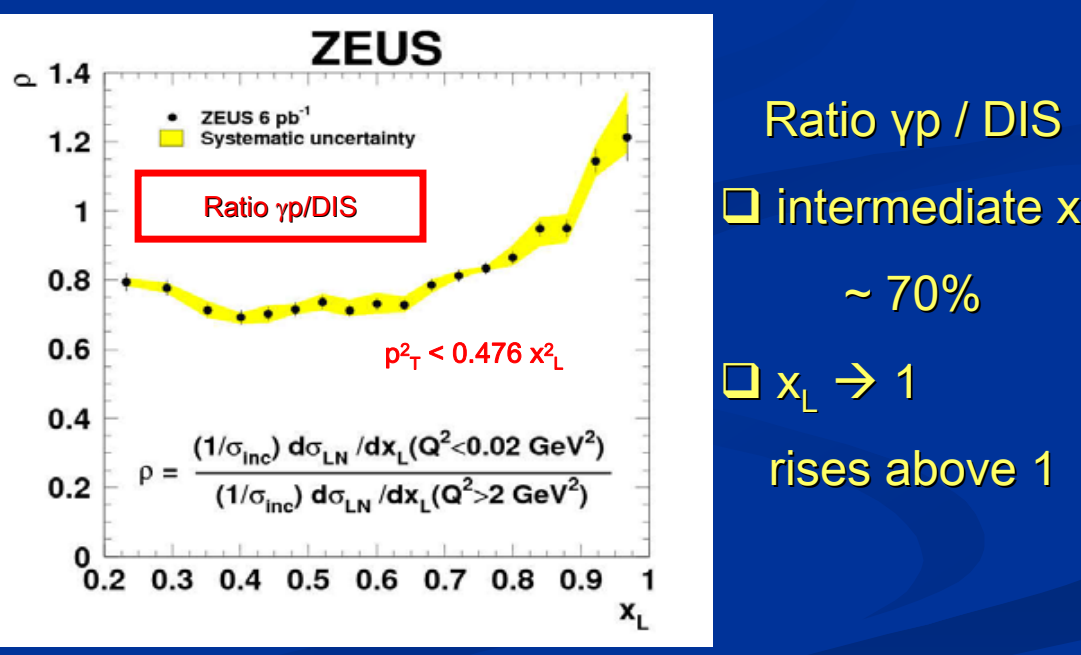

Consistent with absorption

- $\pi-n$ separation decreases at low $X_{L}$ 


\section{LN: $\mathrm{Q}^{2}$ dependence of $\mathrm{p}^{2} \mathrm{~T}$ slopes}

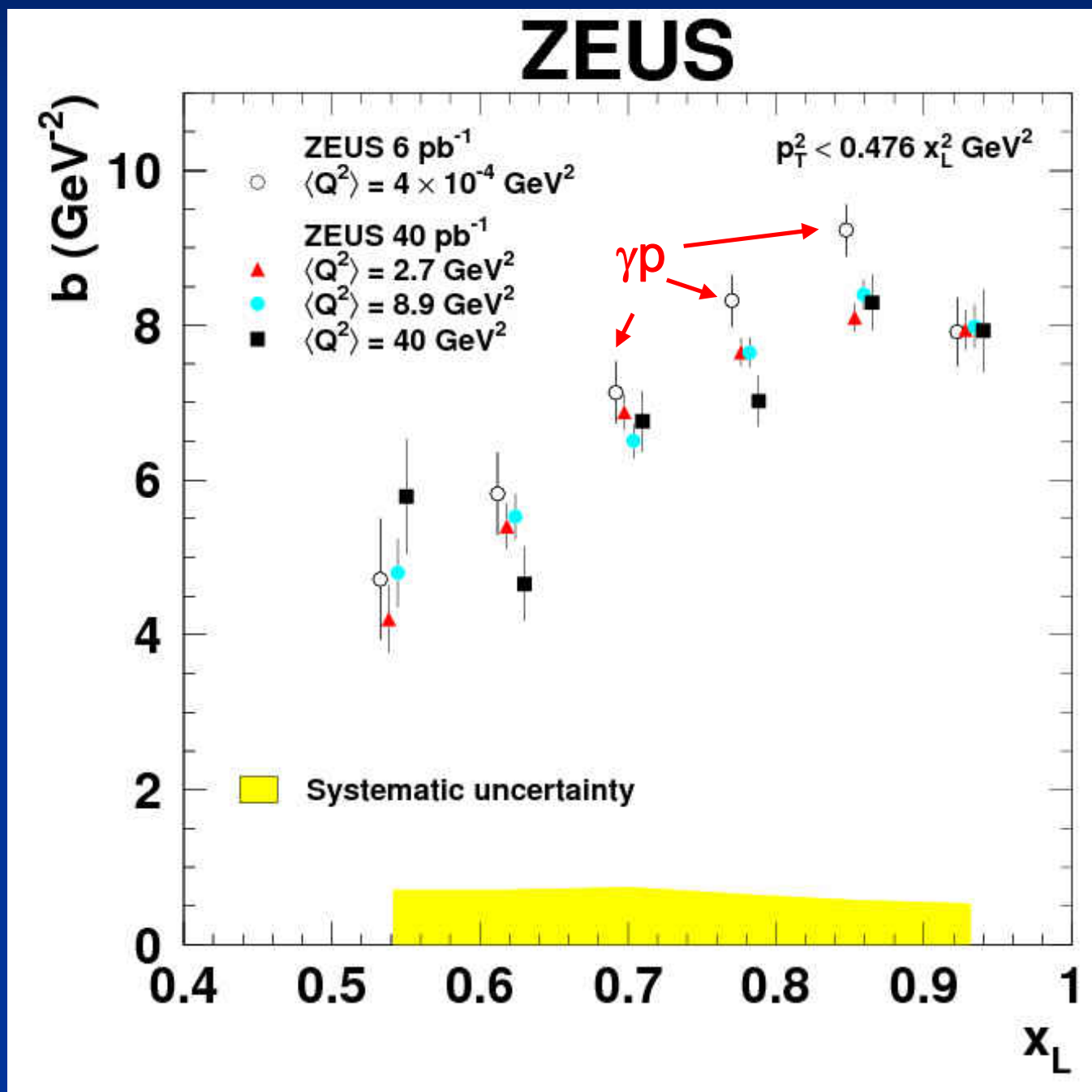

$>$ DIS - slopes:

no $\mathrm{Q}^{2}$ dependence

$>\gamma p$ - slopes:

higher in $0.6<x_{L}<0.9$

\section{Consistent with absorption}

Large $p_{T} \rightarrow$ small $r_{\pi n} \rightarrow$ more abs.

$\rightarrow$ Fewer $\mathrm{LN}$ at high $\mathrm{P}_{\mathrm{T}}$

$\rightarrow$ Steeper slope 


\section{Comparison to models}




\section{Leading Protons - DIS}

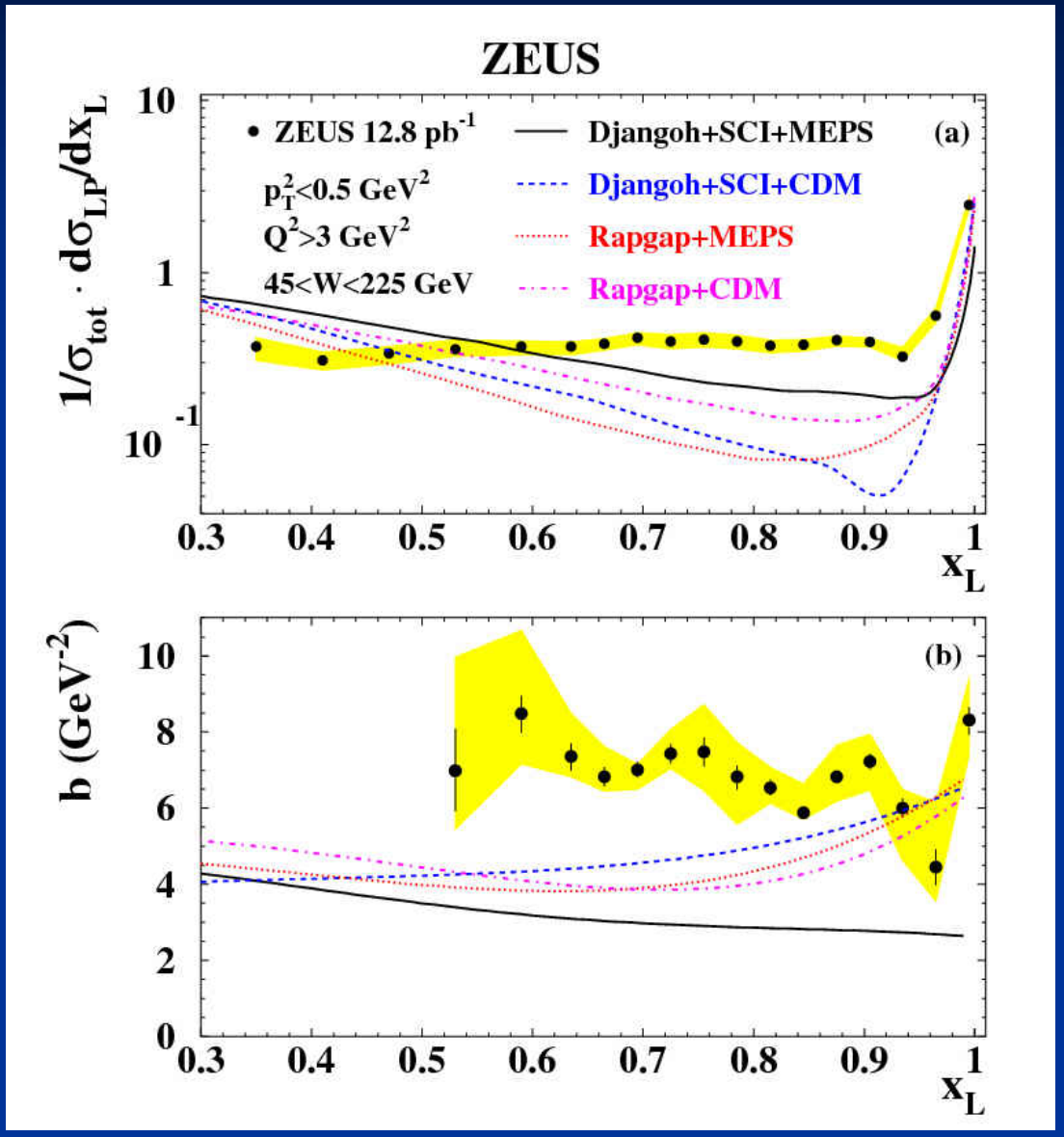

Standard fragmentation MC models fail to describe the data out of the diffractive peak DJANGOH+SCI+MEPS ok b( $\left.x_{L}\right)$ shape
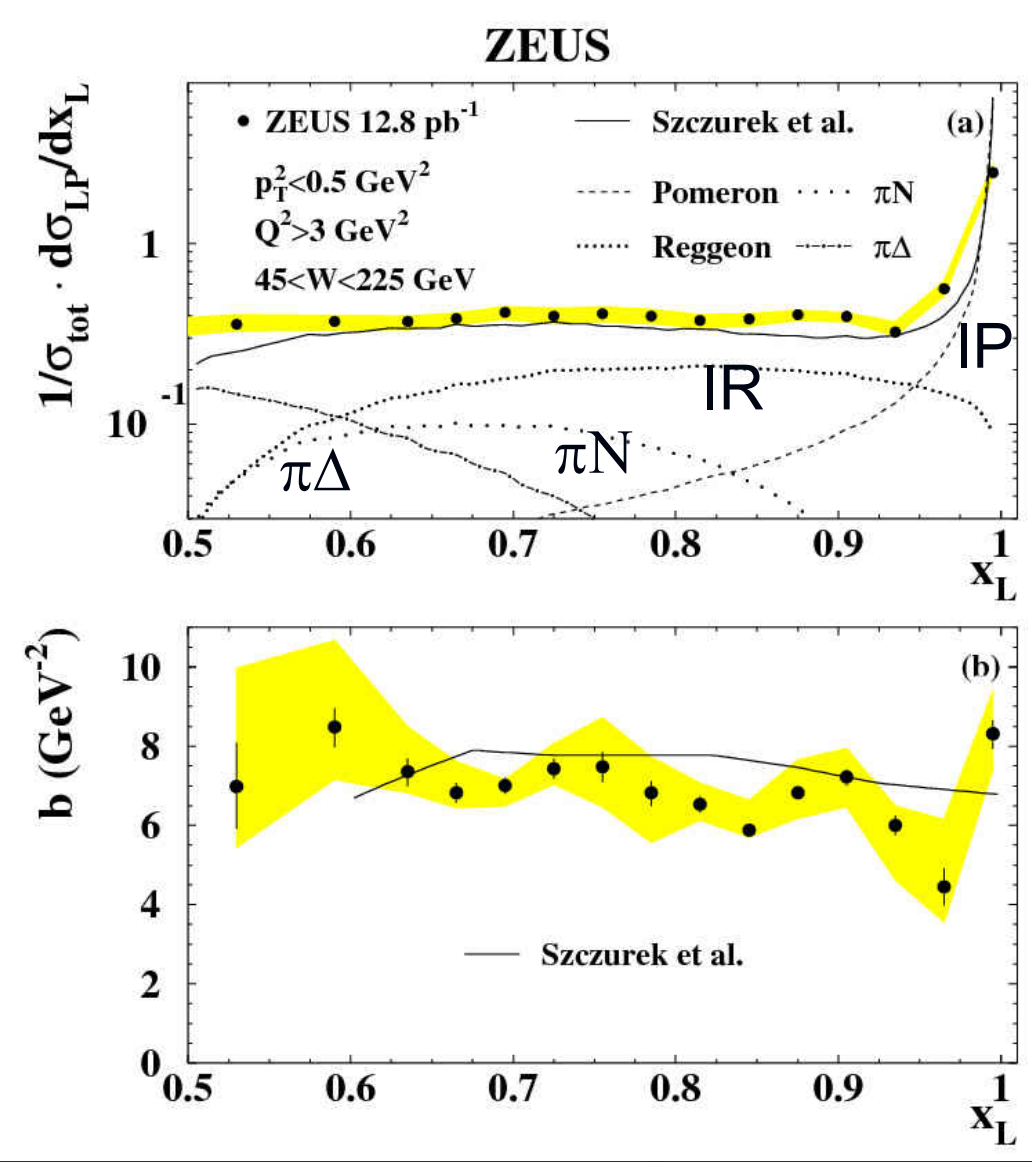

Reasonable description by $\pi$, IR, IP exchange models

Isoscalar IR dominates at intermediate $x_{L}$ 


\section{Leading Neutrons - DIS MC models}

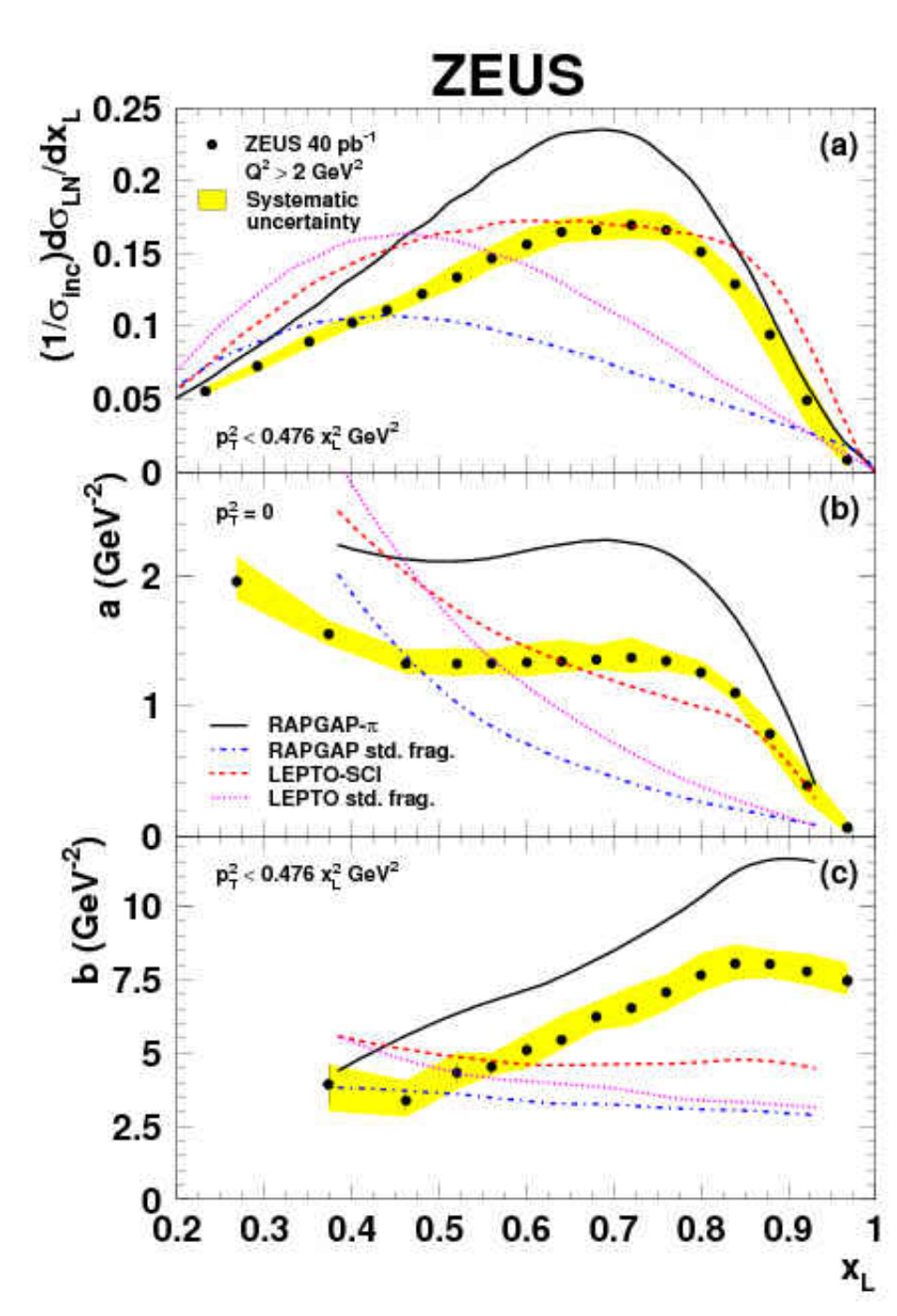

MC models generally fail to reproduce all the features of the data

$\square$ Standard Fragmentation models: understimate the neutron yield and the $X_{L}$ dstribution predicts too many at low $X_{L}$

$\square$ RAPGAP: St. Fragm. $+\pi$-xchng $\rightarrow$ shapes $~ O K$

$\square$ LEPTO+SCl: $x_{\text {L }}$ shapes $\sim$ ok, yield $\sim$ ok, not slopes 


\section{LN: DIS - OPE Models}

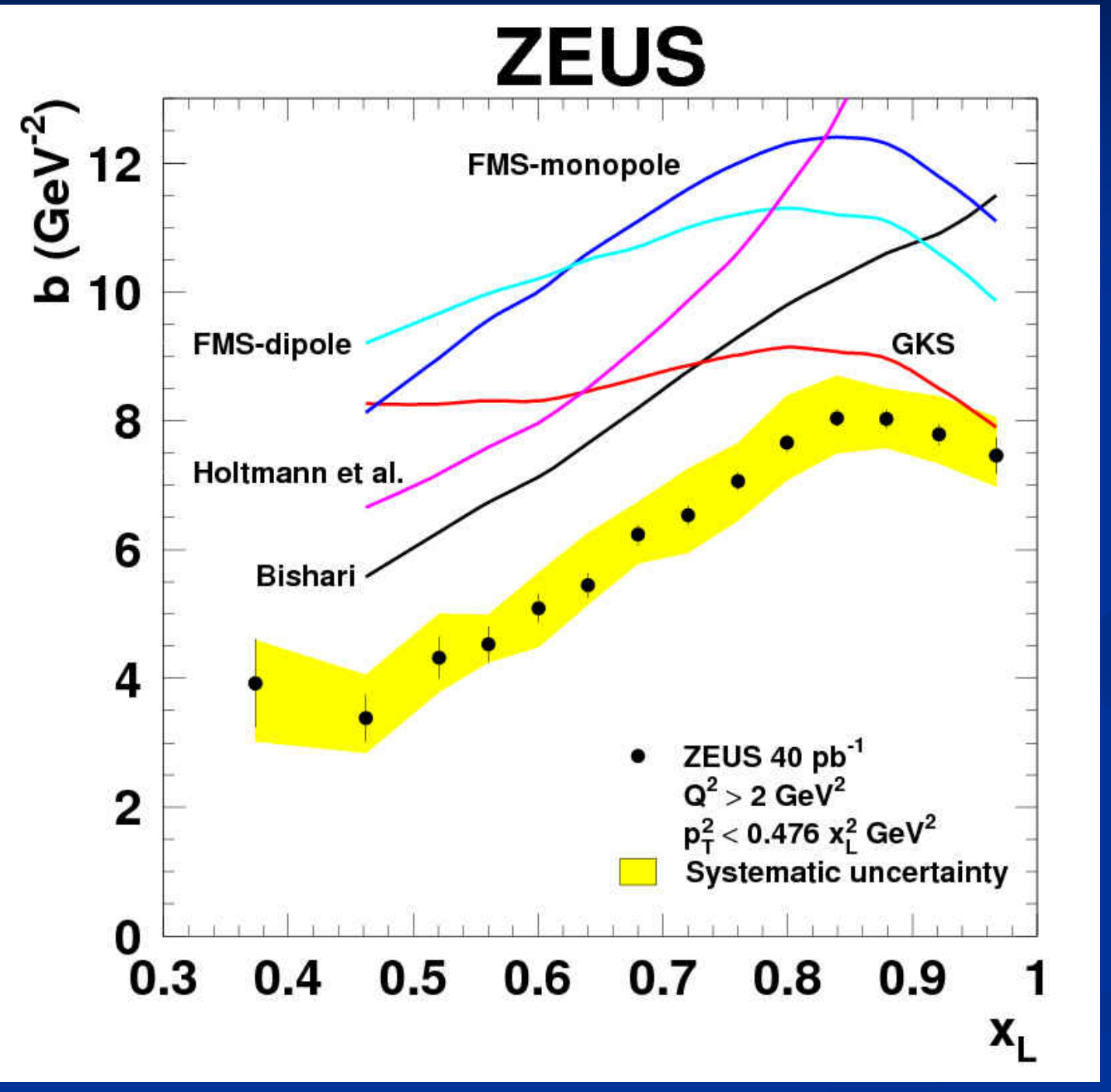

$$
\begin{gathered}
\frac{d \sigma_{e p \rightarrow e n X}}{d x_{L} d p_{T}^{2}}=f_{\pi}\left(x_{L}, p_{T}^{2}\right) \times \sigma_{e \pi}\left(s_{e \pi}\right) \\
f_{\pi} \propto \frac{-t}{\left(t-m_{\pi}^{2}\right)}\left(1-x_{L}\right)^{1-2 \alpha(t)}\left[F\left(x_{L}, t\right)\right]^{2}
\end{gathered}
$$

$\mathrm{p}_{\top}{ }^{2}$ dependence from flux factor

Models shown differ by $\pi-f l u x$ parametrization

Reasonable agreement in shape, not in rate

$\mathrm{p}_{\mathrm{T}}{ }^{2}$ slope (not shown) too high: $\pi$-xchng alone not enough 
LN: DIS - absorption, rescattering + additional IR

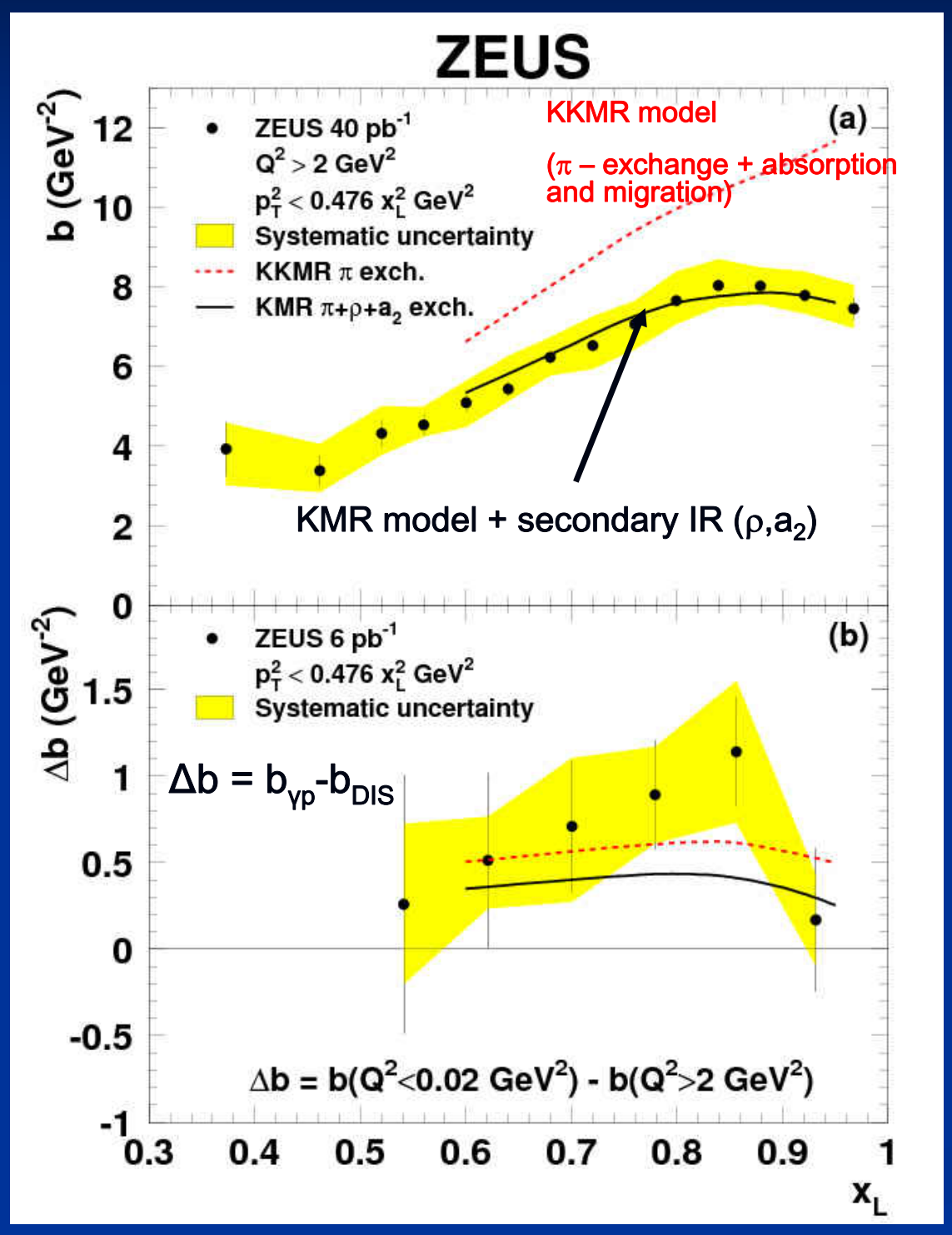

$$
\begin{gathered}
\text { DIS - Slopes } \\
\text { (K)KMR model }
\end{gathered}
$$

Absorption \& migration included in the predictions: not sufficient to describe the slopes

Good description of data when additional IR included 


\section{LN: Photoproduction $-x_{L}$}

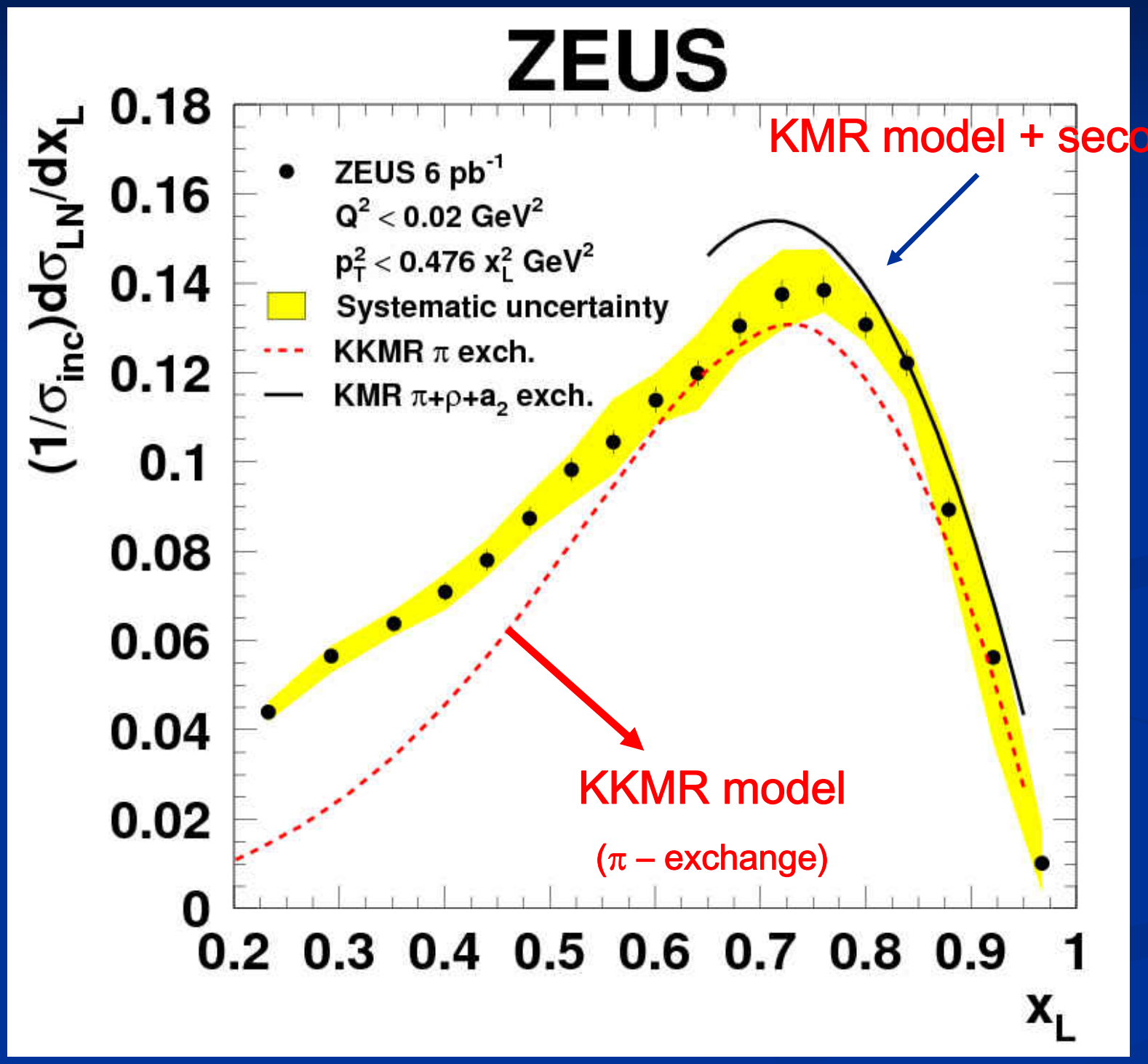

Pure $\pi$-xch. + inclusion migrations in $x_{L}$ and $p^{2}{ }_{T}$ after rescattering

Reasonable description of shape and normaliz.

Add ( $r$,a2)-xch: again reasonable agreement in $y p$ 


\section{LN : comparison of $x_{L}$ yields: $\gamma p / D I S$}

\section{ZEUS}

ㄷ 1.4

- ZEUS $6 \mathrm{pb}^{-1}$

$1.2 \quad \mathrm{p}_{\mathrm{T}}^{2}<0.476 \mathrm{x}_{\mathrm{L}}^{2} \mathrm{GeV}^{2}$

Systematic uncertainty

-.- D' Alesio and Pirner

$1-$ D' Alesio and Pirner $\times\left(1-\mathrm{x}_{\mathrm{L}}\right)^{-0.13}$

-. NSZ

$\cdots \quad N S Z \times\left(1-x_{L}\right)^{-0.13}$

0.8

0.6
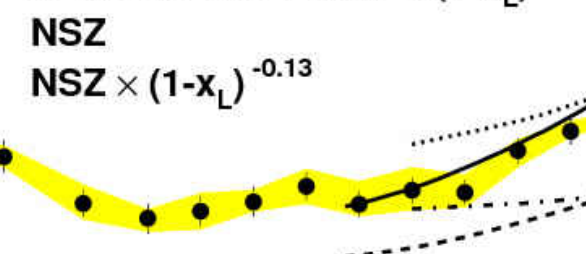

0.4

$0.2 \rho=\frac{\left(1 / \sigma_{i n c}\right) d \sigma_{L N} / d x_{L}\left(Q^{2}<0.02 G e V^{2}\right)}{\left(1 / \sigma_{i n c}\right) d \sigma_{L N} / d_{x_{L}}\left(Q^{2}>2 G e V^{2}\right)}$

0

$\begin{array}{lllllllll}0.2 & 0.3 & 0.4 & 0.5 & 0.6 & 0.7 & 0.8 & 0.9 & 1\end{array}$ $\mathbf{X}_{\mathrm{L}}$
Models: OPE + absorption

$\sigma_{\gamma \pi} \propto s_{\gamma \pi}^{\lambda}=\left[\left(1-x_{L}\right) \times W_{\gamma p}{ }^{2}\right]^{\lambda}$

ZEUS $\frac{\sigma_{\gamma \pi}}{\sigma_{\gamma^{*} \pi}}=\left(1-X_{L}\right)^{\Delta \lambda}=\left(1-X_{L}\right)^{-0.13}$

( different cms energy dependence)

Good agreement with the data 


$$
\begin{aligned}
& \text { LN + dijets in } \\
& \text { photoproduction }
\end{aligned}
$$




\section{Leading $n$ in $\gamma p+$ dijets}

Absorption effects seen going from hard $\rightarrow$ soft scale

High $\mathrm{Q}^{2} \rightarrow$ Low $\mathrm{Q}^{2} \rightarrow \gamma \mathrm{p}$

Reintroduce a hard scale in yp

\section{Still absorption?}

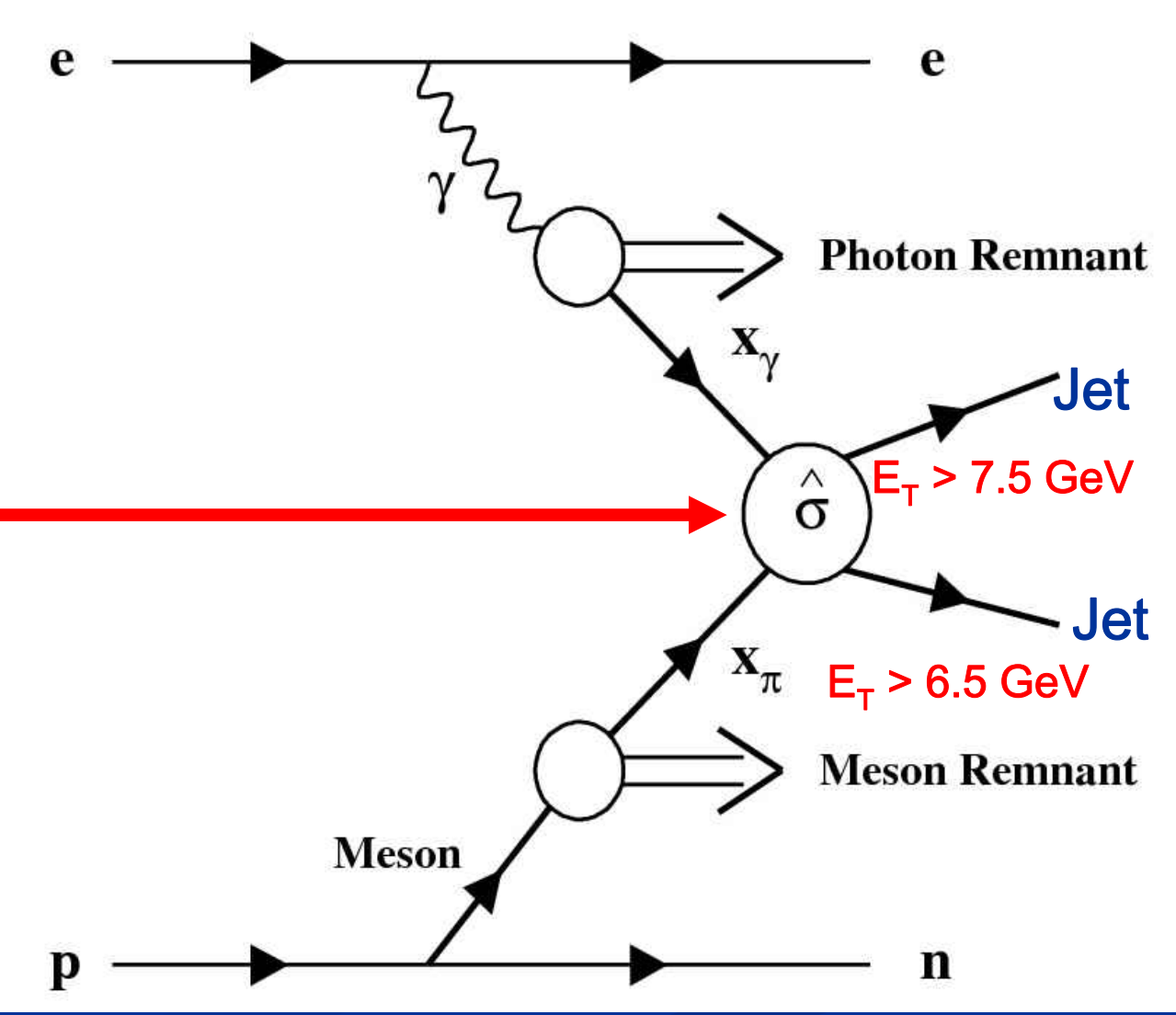




\section{$L N+j j(\gamma p)$ vs LN (DIS)}

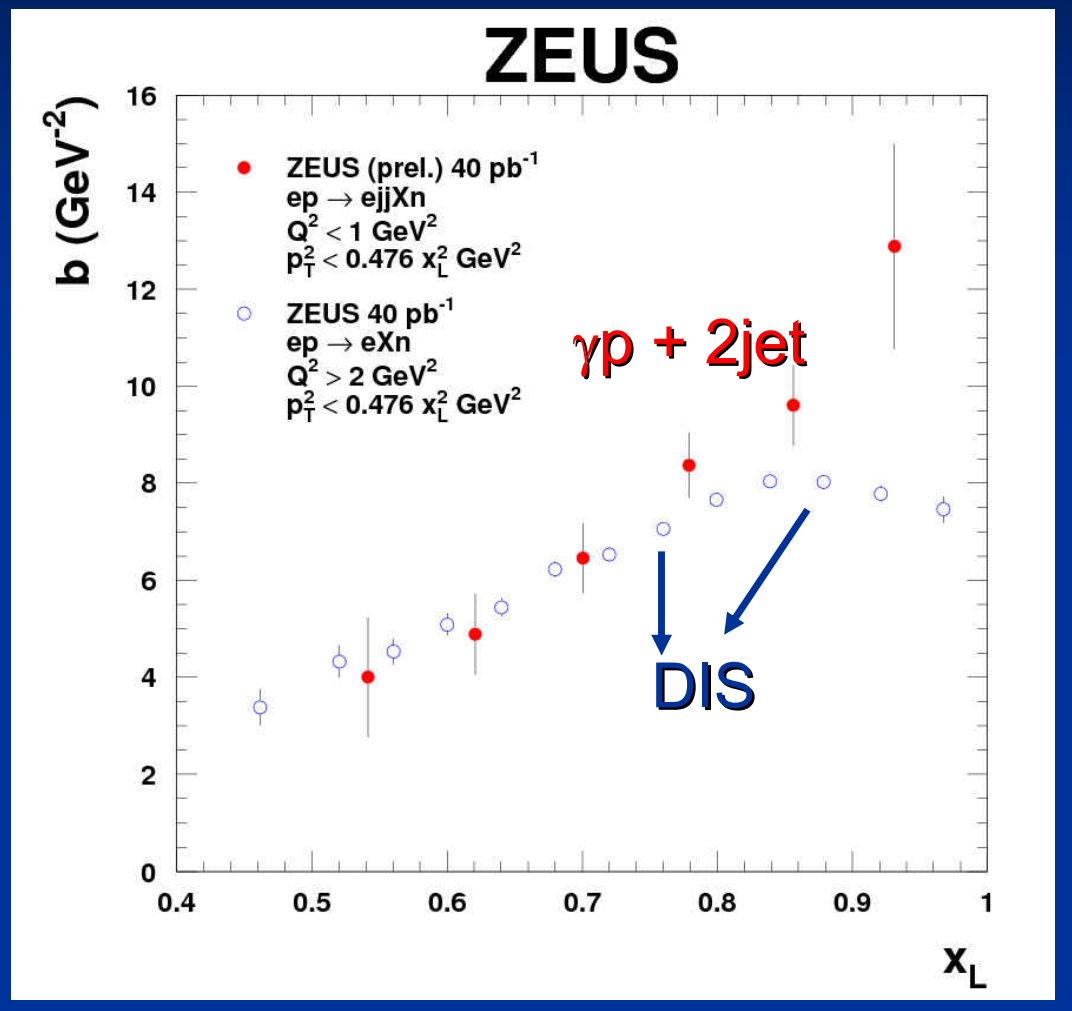

b-slopes similar in magnitude and shape in DIS and $\gamma p+d i j e t$

$\rightarrow$ Same production mechanism

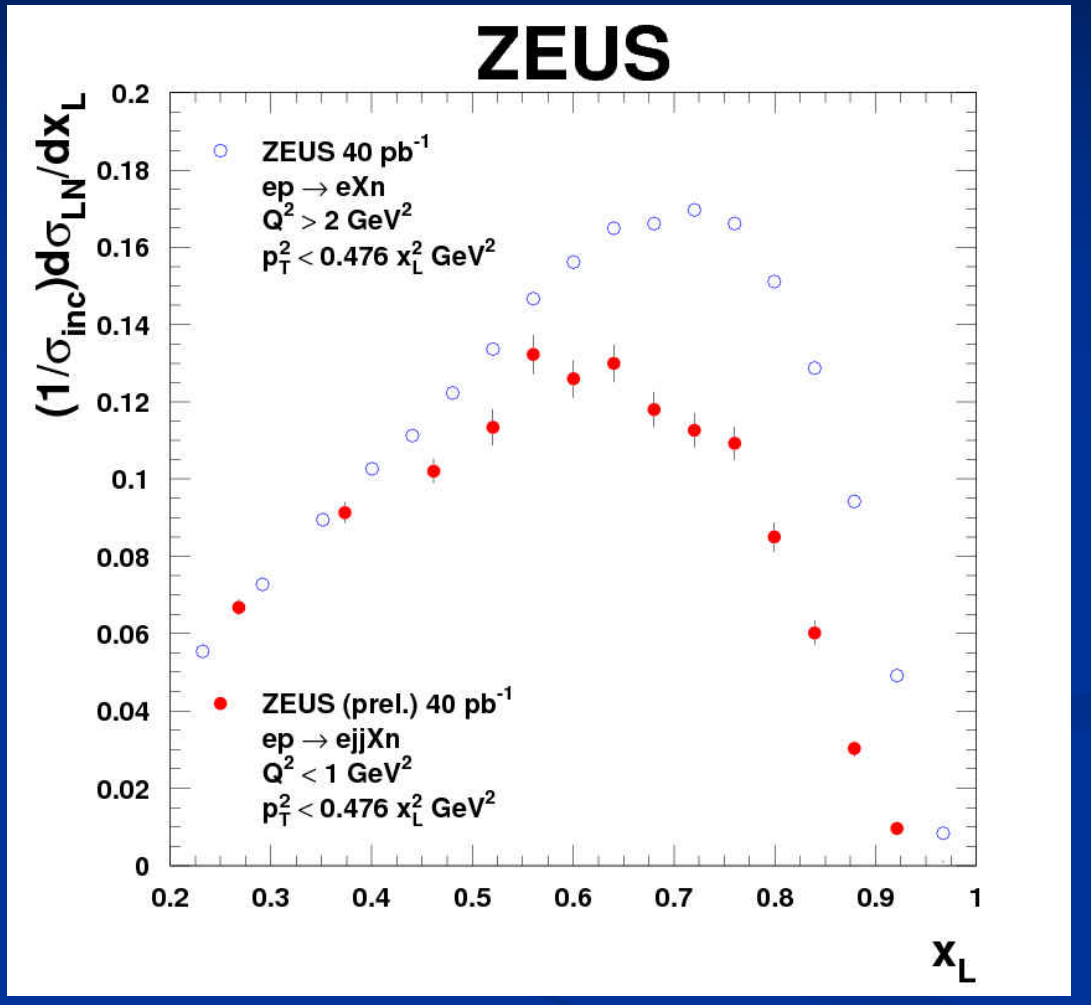

$\square$ yp without jets: suppression at low $X_{L}$

$\square$ yp with jets: suppression at high $x_{L}$

Not yet firm conclusions on absorption 


\section{Summary}

- Recent ZEUS data on LB production in DIS, yp, yp+dijets as a tool to study hard $\leftrightarrow \rightarrow$ soft physics

- Observation of factorization breaking effects going from hard to soft scales, mostly in LN

- Standard fragmentation MC-models fail to describe the data: improvement with particle-exchange implemented

- LP: needs isoscalar IR contributions to explain the data

- LN: pure p exchange not sufficient (slopes not described)

- Recent calculations with $\pi$ exchange and absorption + migration corrections improve the agreement in shape and magnitude of the $x_{L}$ spectra

- Additional exchanges $\left(\rho, a_{2}\right)$ improve further

- yp + hard jets: more work needed 


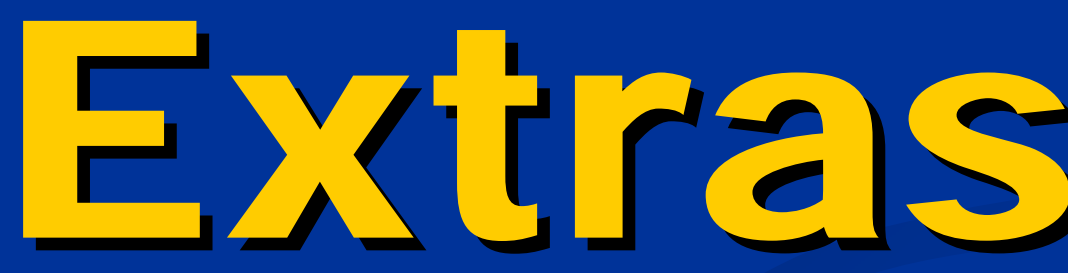




\section{Additional corrections}

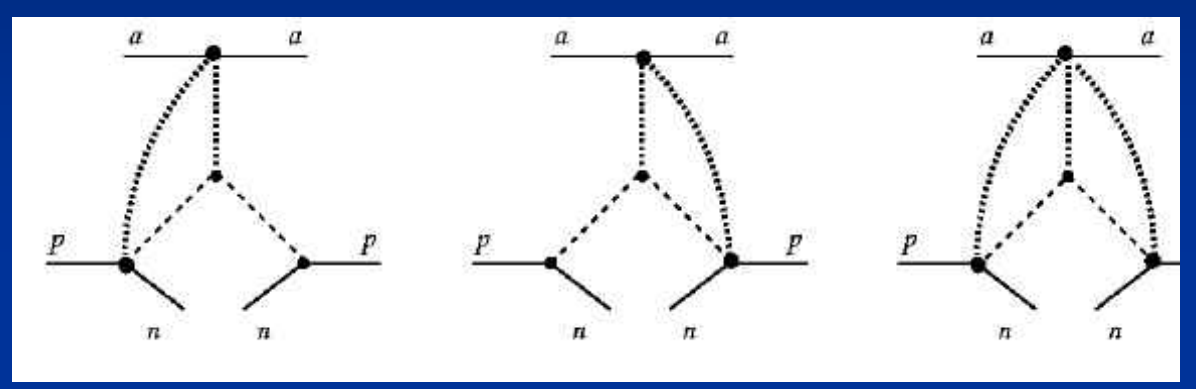

Nikolaev, Speth and Zakharov (NSZ)

(hep-ph/9708290)

Corrections due to re-scattering processes via additional IP exchanges

$(\rightarrow$ Uncertainties in $\pi$ structure function extraction )

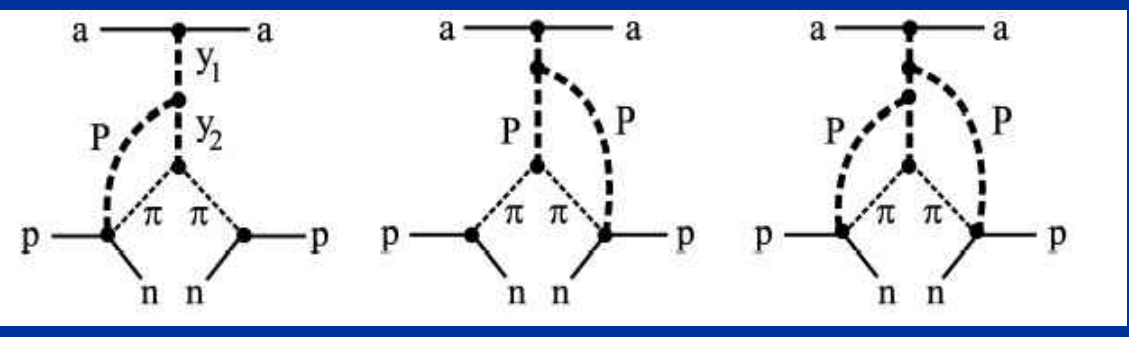

(Kaidalov,) Khoze, Martin, Ryskin (KKMR)

(hep-ph/0602215, hep-ph/0606213)

Enhanced absorptive corrections, calculation of migrations, inclusion of exchange of $\rho$ and $a_{2}$ ( $\rightarrow$ different $x_{L} \& p_{T}$ dependences) 


\section{Acceptance}

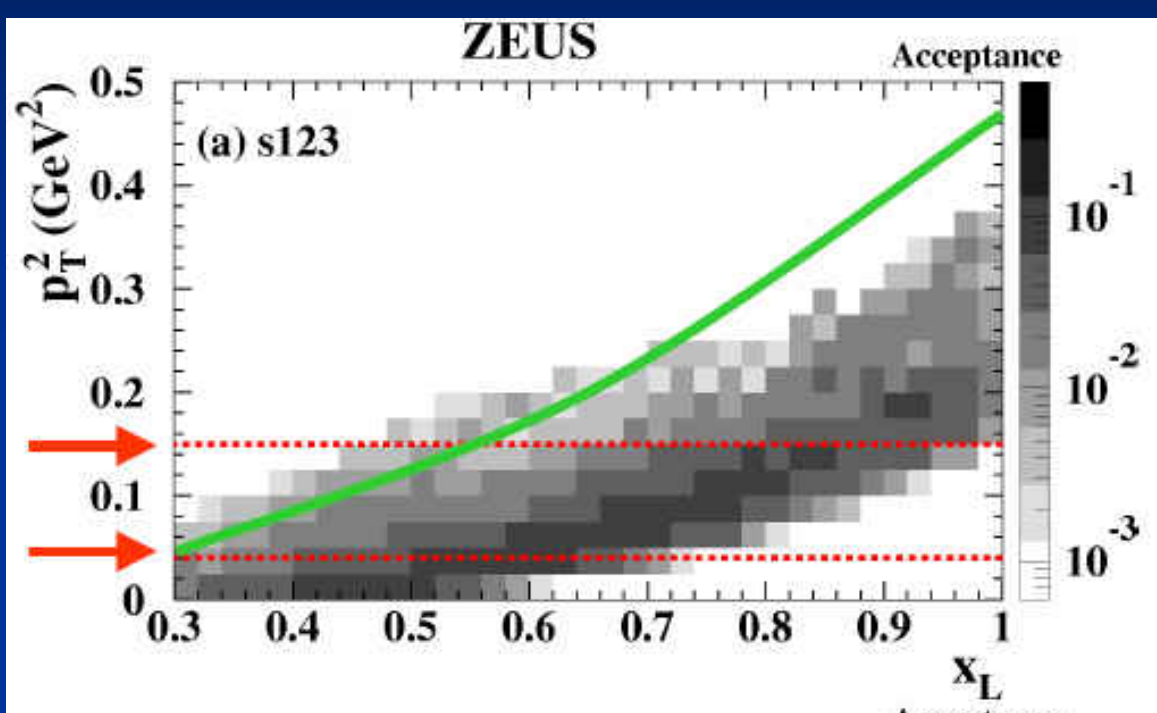

LPS

Acceptance limited by beam elements and detector size

S1-S2-S3 and S4-S5-S6 are independent spectrometers: very small overlap

Accepted $p_{T}$ range varies with $x_{L}$ $x_{L}>0.6 \rightarrow p^{2}{ }_{T}<0.5 \mathrm{GeV}^{2}$

$<$ Acceptance $>15 \%$

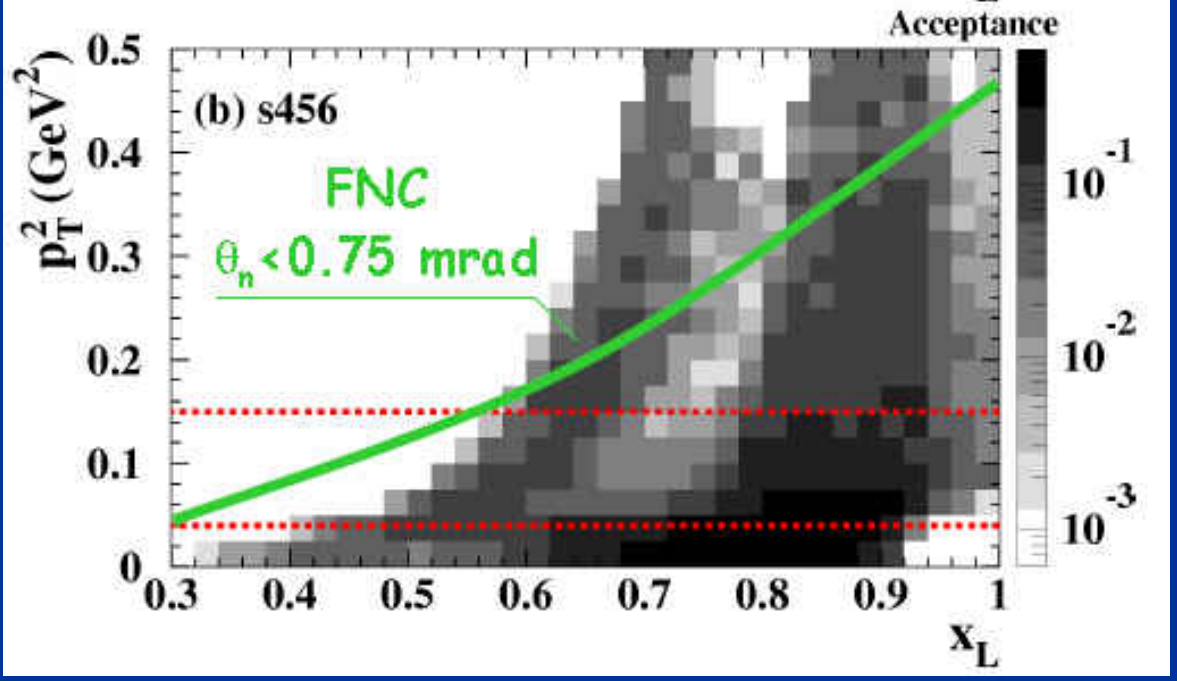

FCN

Restricted to $\Theta_{\mathrm{n}}<0.75 \mathrm{mrad}$ $p_{T}$ range increases with $x_{L}$ For $\mathrm{x}_{\mathrm{L}}>0.6, \Theta_{\mathrm{n}}<0.75 \mathrm{mrad}$ $<$ Acceptance $>25 \%$ 


\section{Slopes and intercepts}

Different trend. Similar around $x_{L} \sim 0.7$

$$
\frac{1}{\sigma_{\text {inc }}} \frac{d^{2} \sigma_{L B}}{d x_{L} d p_{T}^{2}}=a\left(x_{L}\right) \cdot e^{-b\left(x_{L}\right) p_{T}^{2}}
$$

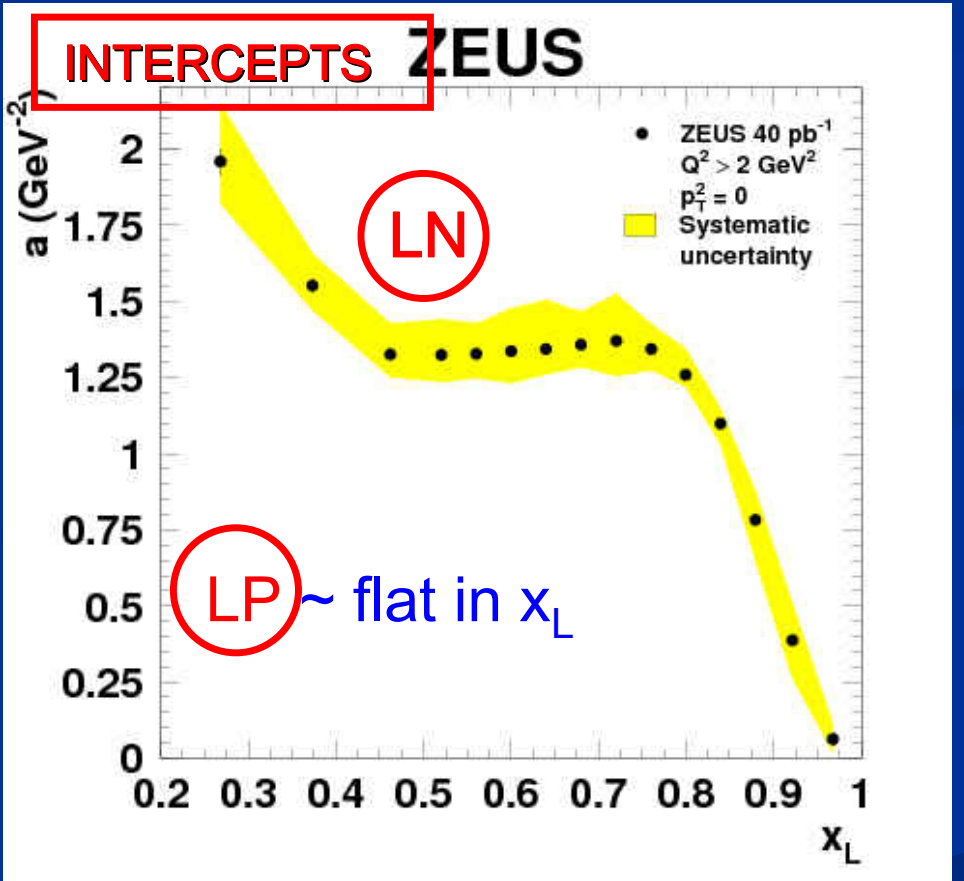

LN falls with $x_{L}$

Flat for $0.4<x_{L}<0.8$ 


\section{Fracture Functions approach}

\section{L.Trentadue, G.Veneziano}

Phys. Lett. B323, 201 (1994)

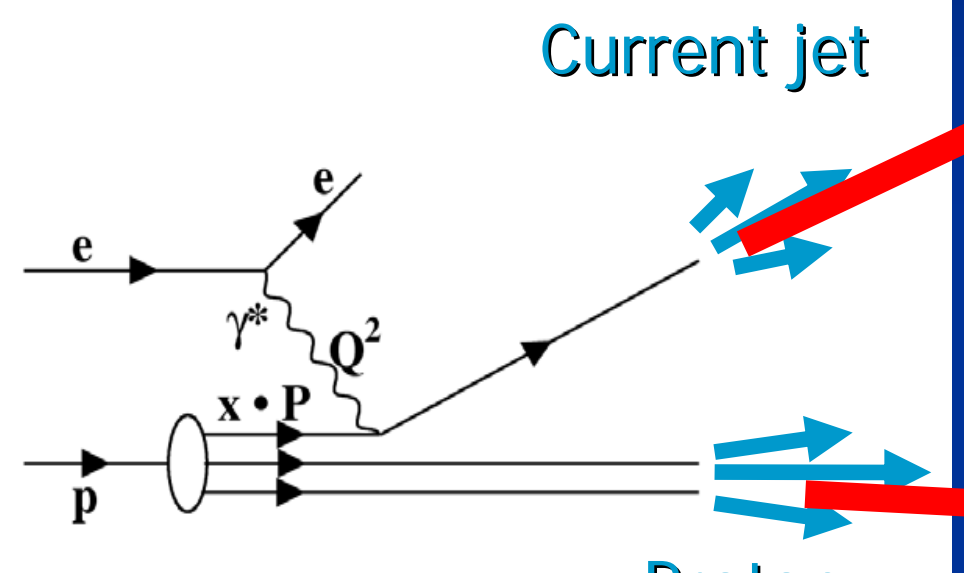

Proton

remnant
Production in the current region

$$
\sigma_{\text {current }} \square \sum_{q} c_{q} f_{q / p}(x) \underset{\text { Fragmentation function }}{D_{h / q}\left(x_{L}\right)}
$$

Production in the target region: F.F.

$$
\sigma_{\text {target }} \square \sum_{q} c_{q}(1-x) M_{q, h / p}\left[x,(1-x) x_{L}\right]
$$

$$
\sigma=\sigma_{\text {current }}+\sigma_{\text {target }}
$$

- F.F. are non-perturbative universal

Dynamical models allow to link them to concepts like structurefunctions of exchanged objects (e.g. IR trajectories) functions (absorb collinear singularities at LO in $\alpha_{s}$ )

- Q2 dependence governed by evolution equations 